\title{
AVALIAÇÃO DA LESÃO ISQUÊMICA NORMOTÉRMICA DO FÍGADO: PAPEL DA OCLUSÃO DO DUCTO BILIAR PRINCIPAL E DA N-ACETILCISTEÍNA
}

\section{EVALUATION OF THE NORMOTHERMIC ISCHEMIC LIVER INJURY: THE ROLE OF MAIN BILIARY DUCT OCCLUSION AND N-ACETYLCYSTEINE}

\author{
Susi Heliene Lauz Medeiros, TCBC-RS' ; Edna Frasson de Souza Montero, TCBC-SP²; \\ Lígia Ferreira Gomes ${ }^{3}$; Murched Omar Taha, TCBC-SP²; \\ Virgínia Berlanga Campos Junqueira ${ }^{4}$; Manuel de Jesus Simões ${ }^{5}$
}

\begin{abstract}
RESUMO: Objetivo: Estudar o efeito da N-acetilcisteína (NAC) na isquemia hepática. Método: Trinta e oito ratos machos EPM1 Wistar foram distribuídos em quatro grupos. Nos Grupos 1 e 2 foi realizado 30 min de clampeamento do hilo hepático, e nos Grupos 3 e 4 os animais foram submetidos a 30 minutos de isquemia sem clampleamento do ducto biliar. Os animais dos Grupos 2 e 4 receberam 150mg.Kg-1 de NAC, endovenoso, 15 minutos antes do procedimento. Colheu-se sangue antes do procedimento e após o clampeamento do pedículo para a dosagem enzimática. Amostras de fígado foram coletadas para dosagem de glutationa, microscopia óptica e eletrônica. No estudo estatístico aplicaram-se testes não paramétricos, $\mathrm{p} \leq 0,05$. Resultados: $\mathrm{O}$ aumento das enzimas foi menor quando se administrou NAC, sendo semelhante na ausência do clampeamento da via biliar. À microscopia óptica houve diferença significante dos grupos S/NAC X C/NAC, mostrando que o grupo C/NAC manteve a arquitetura do parênquima durante a isquemia, independente do clampeamento do ducto biliar. Na microscopia eletrônica os grupos C/NAC e os sem clampeamento do ducto biliar apresentaram arquitetura celular preservada. A NAC não alterou a relação de glutationa reduzida/ glutationa oxidada (GSH/GSSG). Conclusões: A NAC é capaz de proteger o parênquima hepático durante a isquemia normotérmica e propõe-se que o mecanismo seja por reação direta da NAC com o óxido nítrico (NO) (Rev. Col. Bras. Cir. 2005; 32(4): 168-172).
\end{abstract}

Descritores: Acetilcisteína; Isquemia; Fígado; Ratos Wistar.

\section{INTRODUÇÃO}

Durante a interrupção do aporte sangüíneo para um tecido promove-se hipóxia das células, iniciando-se uma seqüência de eventos bioquímicos, quais sejam a parada do funcionamento da cadeia respiratória ${ }^{1}$. A manobra de Pringle tem sido empregada pelos cirurgiões nas ressecções e traumas hepáticos, promovendo uma isquemia durante o ato operatório. Dependendo do tempo de oclusão do pedículo hepático ocorre lesão tecidual que evolui à insuficiência hepática ${ }^{2}$. A isquemia hepática total produzida por esta manobra tem sido utilizada nos estudos em animais de experimentação associada com a derivação vascular, no intuito de prevenir os eventos patológicos em outras áreas, que advêm dessa isquemia, tais como a congestão esplâncnica e a isquemia intestinal, com conseqüente endotoxemia, que evolui com o choque e a morte do animal ${ }^{1,3}$.

Na lesão hepática isquêmica é importante salientar o componente da estase biliar, além da oclusão vascular, no emprego da manobra de Pringle ${ }^{3}$, uma vez que os sais biliares alteram a permeabilidade da membrana celular causando dano ao parênquima hepático ${ }^{4,5}$. Desta forma, es- clarecendo-se os mecanismos de ocorrência da lesão, podese alterar sua evolução empregando estratégias de proteção ao órgão. Destaca-se o emprego de substâncias que, por diferentes mecanismos de ação, promovem uma redução do dano celular, seja atuando como antioxidante ou minimizando a ação dos produtos decorrentes da fase isquêmica ${ }^{6}$. Estas substâncias agem diminuindo a concentração ou neutralizando as espécies reativas de oxigênio e nitrogênio, ou por inibirem a sua formação ou por facilitarem o seu desaparecimento ${ }^{7}$.

Entre os antioxidantes, a glutationa, que é um tiol de baixo peso molecular, está descrita como efetiva na fase de isquemia. Outro tiol de baixo peso molecular, a $\mathrm{N}$-acetilcisteína (NAC) tem seu uso terapêutico consagrado na hepatite tóxica pelo acetaminofen. Nesta condição, a NAC protege o fígado por induzir um aumento da glutationa hepática, que serve como substrato para a inativação e conjugação de substâncias estranhas ao organismo ${ }^{8}$. Ademais, a NAC tem sido empregada na prática clínica em situações de instabilidade hemodinâmica, a fim de proteger os tecidos, quais sejam, pulmonar, cardíaco, renal e hepático, que são comprometidos pelo fenômeno de isquemia seguida de reperfusão ${ }^{9}$. A NAC

\footnotetext{
1. Professor Adjunto do Departamento de Cirurgia da FURG.

2. Professor do Departamento de Cirurgia da UNIFESP-EPM.

3. Professor Adjunto do Departamento de Análises Clínicas e Toxicológicas da USP

4. Professor Adjunto do Departamento de Medicina Interna da UNIFESP-EPM.

5. Professor do Departamento de Morfologia da UNIFESP-EPM.
}

Recebido em 15/12/2004

Aceito para publicação em 10/06/2005

Conflito de interesse: nenhum

Fonte de financiamento: nenhuma

Trabalho realizado na Universidade Federal de São Paulo e Fundação Universidade Federal do Rio Grande. 
age como um antioxidante e também está descrito que é capaz de melhorar o fluxo sangüíneo na microcirculação ${ }^{10}$.

O propósito deste estudo foi avaliar a lesão de isquemia hepática normotérmica, verificando a participação da estase biliar e a modulação dessa lesão pela N-acetilcisteína.

\section{MÉTODO}

Foram utilizados 38 ratos, da linhagem EPM-1 Wistar, machos, com peso médio de $280 \mathrm{~g}$, com idade entre três e quatro meses. Os animais foram fornecidos pelo Centro de Desenvolvimento de Modelos Experimentais para Medicina e Biologia da UNIFESP-EPM. A amostra foi distribuída, em quatro grupos experimentais da seguinte forma: grupo 1 (oito animais, veículo- SG 5\%, com 30 min de isquemia); grupo 2 (10 animais, com NAC, com 30 min de isquemia); grupo 3 (10 animais, com NAC, com 30 min de clampeamento das estruturas vasculares, sem a oclusão do ducto biliar) e grupo 4 (10 animais, veículo- SG 5\%, com 30 min de clampeamento das estruturas vasculares, sem a oclusão do ducto biliar).

Para estudar o efeito do clampeamento do ducto biliar foram comparados os grupos 1 e 2 com os grupos 3 e 4, respectivamente.

Após pesagem, os animais foram anestesiados com uma associação de fármacos: xilazina $\left(25 \mathrm{mg} / \mathrm{Kg}^{-1}\right)$ e cetamina ${ }^{3}$ (50 mg / K g ${ }^{-1}$ ) por via intramuscular, na região lateral da pata traseira direita. Considerou-se anestesiado o animal quando ocorreu perda do reflexo córneo-palpebral e ausência do reflexo de retirada ao estímulo doloroso por preensão da pata traseira contra-lateral.

Foi realizada laparotomia mediana e para as dissecções utilizou-se o microscópio cirúrgico no aumento de 10 vezes e instrumental microcirúrgico. Identificou-se a veia cava caudal e isolou-se este vaso abaixo das veias renais. Neste momento, realizou-se a primeira coleta de sangue $(1 \mathrm{~mL})$.

A seguir, a NAC foi administrada na dose de $150 \mathrm{mg} /$ $\mathrm{kg}$ aos animais dos grupos 2 e 4, 15 minutos antes do clampeamento hilar. Procedeu-se a identificação e dissecção do pedículo hepático, com isolamento do ducto biliar. Em seguida posicionou-se um clampe englobando todos os elementos do pedículo hepático, nos animais dos grupos 1 e 2 e excluindo o ducto biliar, nos animais dos grupos 3 e 4 . O tempo de isquemia foi de 30 minutos para todos os animais.

Ao final do tempo de isquemia foi realizada a $2^{\mathrm{a}}$ coleta de sangue e enviado para o laboratório de análises clínicas da Técnica Operatória e Cirurgia Experimental da UNIFESPEPM. Foram determinados os valores da atividade das seguintes enzimas: aspartato aminotrasferase, alanina aminotransferase, desidrogenase lática, e a concentração da bilirrubina total, direta e indireta, por método automatizado (COBAS-MIRA).

A morte dos animais foi produzida por exsangüinação, quando se retirava o fígado do animal ainda anestesiado. $\mathrm{O}$ fígado removido era submetido à cortes de aproximadamente $1 \mathrm{~cm}$ x $1 \mathrm{~cm}$ que foram conservados em solução de formaldeído à $10 \%$, para avaliação à microscopia óptica. O tecido foi submetido às técnicas histológicas e corado pela técnica de hematoxilina-eosina e analisada a presença ou au- sência de congestão da veia centro-lobular, espaço porta e sinusóides hepáticos, bem como a presença de vasodilatação. Os achados foram graduados, de acordo com a sua intensidade, em discreta, moderada e acentuada.

Outros fragmentos de fígado $(0,5 \mathrm{~cm} \times 0,5 \mathrm{~cm})$ foram conservados em glutaraldeído a $4^{\circ} \mathrm{C}$ e encaminhados para a microscopia eletrônica. As alterações do citoplasma, do núcleo e das organelas intra-citoplasmáticas, quais sejam retículo endoplasmático, mitocôndrias e lisossomos no período de isquemia foram analisados, sob o ponto de vista descritivo.

Foram obtidos fragmentos do parênquima hepático e congelados $\mathrm{a}-80^{\circ} \mathrm{C}$, para a dosagem de glutationa. Aproximadamente $0,2 \mathrm{~g}$ do tecido hepático foi homogenizado e submetido à cromatografia líquida de alta resolução (HPLC). Para comparação foram utilizados padrões de glutationa reduzida (GSH) e glutationa oxidada (GSSG) na concentração de $0,1 \mathrm{mM}$.

Para a análise dos resultados foram aplicados os seguintes testes:

1. Teste de Wilcoxon para comparar, os valores bioquímicos pré e pós-operatórios.

2. Teste de Mann-Whitney para comparar as mesmas dosagens bioquímicas, entre os dois grupos de estudo.

Fixou-se em 0, 05 ou 5\% (a $\leq 0,05)$ o nível de rejeição da hipótese de nulidade, assinalando-se com um asterisco (*) os valores significantes.

\section{RESULTADOS}

O aumento das enzimas aspartato aminotransferase, alanina aminotransferase e desidrogenase lática , bem como a concentração de bilirrubina total, foi menor quando se administrou a NAC, sendo semelhante na ausência do clampeamento do ducto biliar.

À microscopia óptica houve diferença significante dos grupos S/NAC X C/NAC, mostrando que o grupo C/NAC manteve a arquitetura do parênquima durante a isquemia, independente do clampeamento do ducto biliar. Na microscopia eletrônica os grupos G2 (C/NAC e com clampeamento da tríade portal) e o G4 (C/NAC e sem clampeamento do ducto biliar) apresentaram arquitetura celular preservada sem demonstrar diferença significante.

A NAC não alterou a relação de glutationa reduzida/ glutationa oxidada (GSH/ GSSG).

\section{DISCUSSÃO}

O modelo de isquemia total sem derivação portosistêmica no fígado, durante 30 minutos, simula a manobra de Pringle ${ }^{3}$, utilizada nas ressecções hepáticas e nos transplantes. Entretanto, variações do procedimento operatório como a oclusão das estruturas vasculares sem o clampeamento do ducto biliar, ou a utilização de substâncias protetoras da lesão de isquemia, como a NAC, tem efeitos no sucesso do procedimento e no pós-operatório. Isto ocorre porque a lesão de isquemia evolui à morte celular e perda da função do órgão, decorrente do colapso na produção de energia pela cadeia respiratória, mobilização dos depósitos de cálcio e perda da função das membranas ${ }^{2,10}$. 


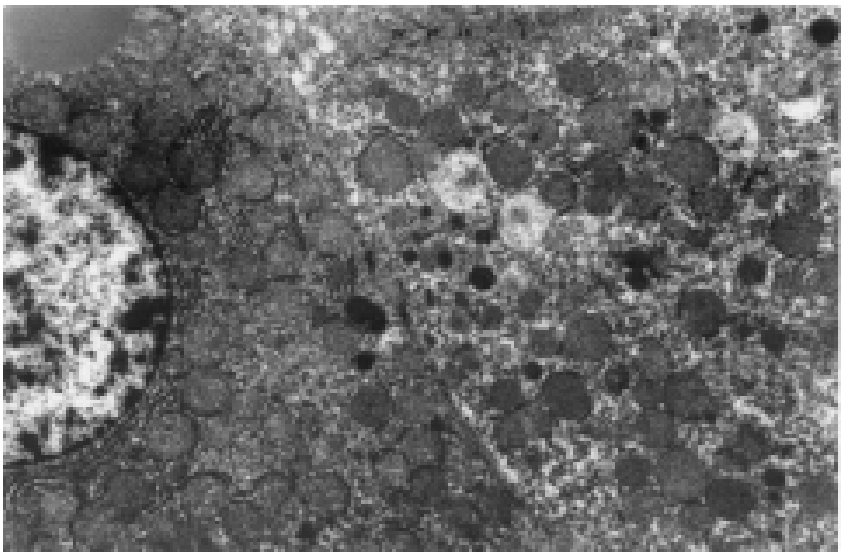

Figura 1 - Eletromicrografia do Grupo 1. Hepatócitos com núcleo rico em heterocromatina, citoplasma com áreas eletrotranslucentes, mitocôndrias mais concentradas e próximas do núcleo, algumas em cristólise, retículo endoplasmático granular e aumento do número de vesículas eletrodensas. X 6800.

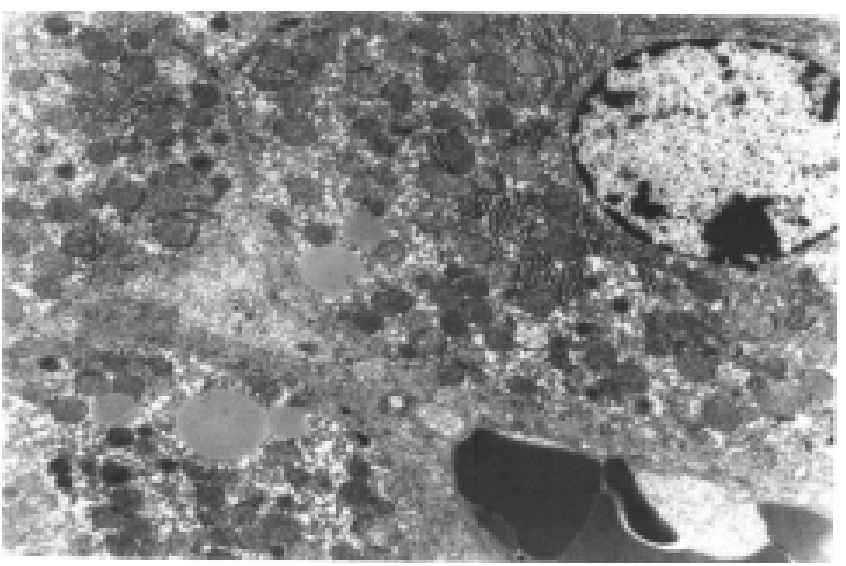

Figura 3 - Eletromicrografia do Grupo 3. Hepatócitos com núcleo rico em heterocromatina, citoplasma com áreas eletrotranslucentes, sugestivas de necrose, mitocôndrias mais concentradas e próximas do núcleo, algumas em cristólise, retículo endoplasmático granular e aumento do número de vesículas eletrodensas. X 6600.

A lesão de isquemia e reperfusão ocorre como um conjunto sequencial de modificações patológicas para o estabelecimento do dano tecidual. Entretanto, tanto os mecanismos envolvidos, quanto as possibilidades de intervenção são diferentes em cada fase. Em particular, a condição tecidual resultante da fase isquêmica interfere na reatividade precoce do tecido à reperfusão, seja pela quantidade de espécies reativas que se formam localmente, seja pela capacidade de eliminação dessas substâncias ou de regeneração do tecido viável remanescente ${ }^{4,7,11}$.

Os resultados apresentados na microscopia óptica mostram que a administração de NAC promove uma preservação morfológica e funcional do tecido durante a fase isquêmica. É possível observar uma manutenção da arquitetura tecidual do fígado, com a veia centro-lobular sem dilatação, capilares sinusóides sem alterações, mostrando uma ação protetora ao tecido na fase isquêmica, corroborando os achados de Sébe ${ }^{11}$. À ultraestrutura, a proteção foi evidenciada por uma menor deterioração do hepatócito, como mostrado nas Figuras 2 e 4.

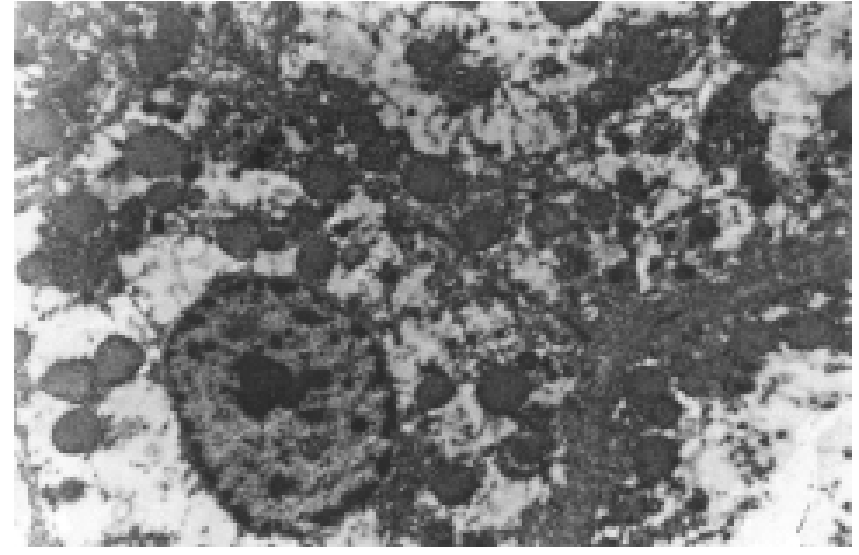

Figura 2 - Eletromicrografia do Grupo 2. Hepatócitos íntegros de forma poliédrica, núcleo esférico e central, rico em eucromatina, citoplasma contendo mitocôndrias, retículo endoplasmático granular e vesículas eletrodensas. X 6800 .

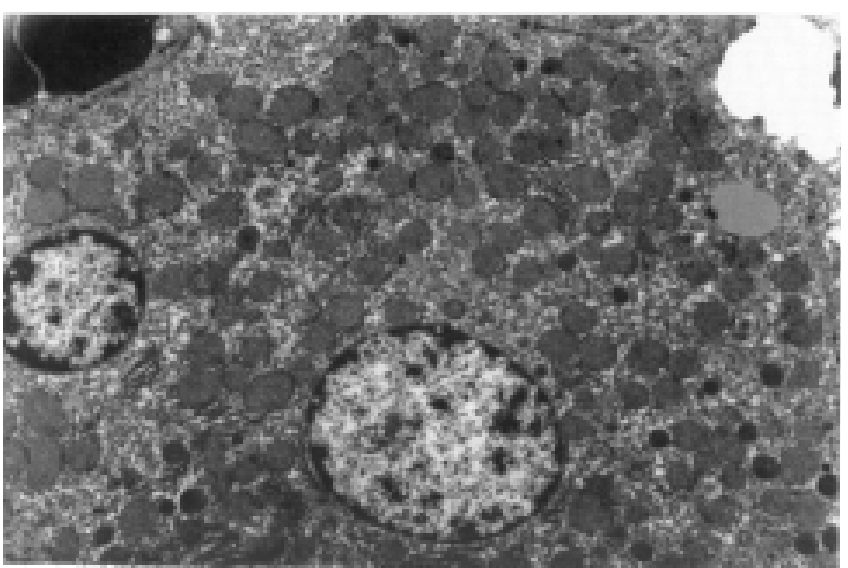

Figura 4 - Eletromicrografia do Grupo 4. Hepatócitos íntegros de forma poliédrica, núcleo esférico e central, rico em eucromatina, citoplasma contendo mitocôndrias, retículo endoplasmático granular e vesículas eletrodensas. X 6600.

Entre as modificações estruturais alvo da proteção pela NAC, destaca-se o aumento do tamanho das mitocôncrias, a destruição das cristas mitocondriais, o aumento do número e volume de lisossomas (Figuras 1 e 3). Este conjunto de alterações é considerado marcador do processo de necrose da célula, responsável pela lise do conteúdo das organelas celulares. A menor destruição celular implica na menor liberação de enzimas celulares na circulação.

Como verificado por Shibayama et al. ${ }^{12}$, encontramos aumento da atividade das enzimas aspartato aminotransferase, alanina aminotransferase e desidrogenase lática. Segundo Shibayama, este aumento relaciona-se com o dano celular hepático.

A oclusão da via biliar pode ampliar o dano, segundo Pastor et al. ${ }^{5}$. Nossos resultados não corroboram esta hipótese, pois não foi possível verificar esta proteção diferencial da NAC nos grupos com e sem clampeamento da via biliar na análise morfológica, na ultra-estrutura (Figura 1 a 4), nos valores de atividade enzimática ou de concentração plasmática de 
bilirrubina . Provavelmente os efeitos da NAC sobre a peroxidação lipídica aumentada na vigência de estase biliar, sugeridos por Pastor ${ }^{5}$, só se reflitam na morfologia em situações de estase crônica. Deve-se considerar também que nos animais com clampeamento vascular, a concentração hepática da NAC pode ser menor, porque a NAC aumenta o fluxo biliar e é excretada pela bile, como observado por Holdiness ${ }^{10}$.

$\mathrm{O}$ efeito da NAC pode ser direto, como um análogo da glutationa tecidual, ou indireto, porque ela é considerada um precursor da glutationa. As medidas de glutationa reduzida e glutationa oxidada permitiram verificar que não houve alterações nas concentrações de glutationa total e reduzida na presença de NAC. Desta forma, confirma-se a hipótese de que a NAC atua diretamente protegendo o dano celular ${ }^{13,14}$.

Seu efeito protetor é atribuído a um efeito antioxidante clássico, pela reação de oxirredução com as espécies reativas de oxigênio produzidas na fase de reperfusão. No presente estudo foi documentada a prote- ção pela NAC na lesão por isquemia que não pode ser explicada pela ação de limpeza (scavenger) de espécies reativas de nitrogênio ou de produtos de degradação de moléculas endógenas por estas espécies, já que nesta fase não se formam quantidades importantes de espécies reativas de oxigênio, pela ausência do substrato ${ }^{13,15-17}$. Os resultados apresentados permitem concluir que a NAC protege o tecido hepático da lesão isquêmica normotérmica, eliminando a congestão e atenuando o dano celular que resulta em comprometimento funcional do órgão. $\mathrm{O}$ uso da NAC durante um período de 30 min de isquemia normotérmica é capaz de reduzir a liberação das enzimas aspartato aminotransferase, alanina aminotransferase e desidrogenase lática e o aumento da concentração tecidual de bilirrubina e leva a uma melhor preservação morfológica do tecido à microscopia ótica e eletrônica. Consideramos, portanto, que pacientes que necessitam ser submetidos à isquemia hepática de forma eletiva possam se beneficiar com o uso desta substância.

\begin{abstract}
Background: The aim of this study was to investigate the effect of N-Acetylcysteine (NAC) on the hepatic ischemia injury. Methods: Thirty eight male EPM-1 Wistar rats were divided in four groups: G1 and G2 with ischemia time of 30 min.; groups 3 and 4 were submitted to 30 min of ischemia and bile duct was not clamped. Animals from groups 2 and 4 received NAC, $150 \mathrm{mg} . \mathrm{Kg}^{-1} \mathrm{bw}$, by IV injection, $15 \mathrm{~min}$. before procedure. Blood samples were collected before and after ischemia and liver function was evaluated by enzymatic measurement. Hepatic samples were processed to GSH/GSSG, light and electronic microscopy evaluation. Non-parametric tests were applied to the statistical analysis ( $p \leq 0.05)$. Results: Enzymatic increase were higher when NAC was absent. There was no protection by NAC when bile duct was absent nor when bile duct was not clamped. Under light microscopy there was significant difference in the groups $S / N A C X C / N A C$, showing that group C/NAC maintained better parenchyma architecture during ischemia time, independent on bile duct clamp. Under electronic microscopy, the groups C/NAC and those without bile duct clamping showed preserved cellular arquitecture. NAC did not alter the relationship between reduced glutathione / oxidated gluthatione (GSH/GSSG).Conclusion: NAC is able to protect hepatic parenquime during normothermic ischemia and we purpose that such mechanism is related to a direct reaction of NAC with nitric oxide $(\mathrm{NO})$.
\end{abstract}

Key words: Acetylcysteine; Ischemia; Liver; Rats, Wistar.

\section{REFERÊNCIAS}

1. Chavez-Cartaya R, Jamieson NV, Ramirez P, et al. Free radical scavengers to prevent reperfusion injury following experimental warm liver ischaemia. Is there a real physiological benefit? Transpl Int. 1999;12(3):213-21.

2. Hannoun L, Borie D, Delva E, et al. Liver resection with normothermic ischaemia exceeding $1 \mathrm{~h}$. Br J Surg. 1993; 80(9):1161-5.

3. Pringle JH. Notes on the arrest of hepatic hemorrhage due to trauma. Ann Surg. 1908;48:541-9.

4. Baron V, Muriel P. Role of glutathione, lipid peroxidation and antioxidants on acute bile- duct obstruction in the rat. Biochim Biophys Acta. 1999;1472(1-2):173-80.

5. Pastor A, Collado PS, Almar M, et al. Antioxidant enzyme status in biliary obstructed rats: effects of $\mathrm{N}$-acetylcysteine. J Hepatol. 1997;27(2):363-70.

6. Goode HF, Webster NR, Howdle PD, et al. Reperfusion injury, antioxidants and hemodynamics during orthotopic liver transplantation. Hepatology. 1994;19(2):354-9.
7. McCord JM. Oxigen-derived free radicals in postischemic tissue injury. New Engl J Med. 1985; 312(3):159-63.

8. Walker MW, Kinter MT, Roberts RJ, et al. Nitric oxide-induced cytotoxicity: involvement of cellular resistance to oxidative stress and the role of glutathione in protection. Pediatr Res. 1995; 37(1):41-9.

9. Tepel M, van der Giet M, Schwarzfeld C, et al. Prevention of radiographic-contrast-agent-induced reductions in renal function by acetylcysteine. New Eng J Med. 2000; 343(3):180-4.

10. Holdiness MR. Clinical pharmacokinetics of $\mathrm{N}$-acetylcysteine. Clin Pharmakcokinet. 1991;20(2):123-34.

11. Sébe AA. Efeitos de diferentes tempos de isquemia no fígado de ratos [dissertação]. São Paulo (SP): UNIFESPEPM, 1999.

12. Shibayama Y, Asaka S, Nishijima A. Mechanism of liver injury following ischemia. Exp Mol Pathol. 1991;55(3):251-60.

13. Vivot C, Stump DD, Schwartz ME, et al. Nacetylcysteine attenuates cold ischemia/reperfusion injury in the isolated perfused rat liver. Transplant Proc. 1993; 25(2):1983-4. 
14. Moncada S, Palmer RM, Higgs EA. Nitric oxide: physiology, pathophysiology, and pharmacology. Pharmacol Rev. 1991; 43(2-3):109-42.

15. Murphy MP. Nitric oxide and cell death. Biochim Biophys. 1999; 1411(2-3):401-14.

16. Zweier JL, Samouilov A, Kuppusamy P. Non-enzymatic nitric oxide synthesis in biological systems. Biochim Biophys Acta.1999;1411(2-3):250-62.
17. Gaston B. Nitric oxide and thiol groups. Biochim Biophys Acta. 1999;1411(2-3):323-33.

Endereço para correspondência :

Susi Lauz

Mal. Deodoro, 521, ap 301.

96211-480- Rio Grande - RS

E-mail: lauz@zipmail.com.br 